\title{
The Pythagorean triples whose hypotenuse and the sums of the legs are squares
}

\author{
PROF. DR. K. RAJA RAMA GANDHI ${ }^{1}$ AND REUVEN TINT ${ }^{2}$ \\ Resource person in Math for Oxford University Press and Professor at BITS-Vizag ${ }^{1}$ \\ Number Theorist, Israel ${ }^{2}$ \\ Email: editor126@gmail.com, reuven.tint@gmail.com
}

\begin{abstract}
More than 370 years ago the famous French mathematician Pierre de Fermat proposed to solve the following problem: to find a Pythagorean triples whose hypotenuse and the sum of the legs were squares, which, despite its simplicity, has been very difficult. Problems associated with its solution involved many mathematicians such as (Leonhard Euler, Joseph-Louis Lagrange, Ljnggren, Wacław Sierpiński and etc.) But in the end it did not reach solution. In our article, solution communicated to obtain the equations giving the required values all elements of the Pythagorean triples in positive integers (natural) are co-prime integers, and provides a second solution of this problem (the values of $\mathrm{x}, \mathrm{y}, \mathrm{z}$ of 45 digits), and some consequences.
\end{abstract}

\section{$\S 1$}

In 1643, Fermat challenged Mersenne to find a Pythagorean triplet whose hypotenuse and sum of the legs were squares. ("very difficult to solve the following problem", Diophantus of Alexandria, "Arithmetica”, Наука, 1974 p. 309) Fermat found the smallest such solution.

$$
\begin{gathered}
x=4565486027761 \\
y=1061652293520 \\
z=4687298610289= \\
=(2165017)^{2} \\
x+y=(2372159)^{2}
\end{gathered}
$$

Problems associated with the solution of this problem, were engaged Euler, Lagrange, Ljunggren and etc. The first person who show how to obtain first solution of the problem, that resulted by Fermat was the Wacław Franciszek Sierpiński. He also solved the problem of obtaining all results that include coprime solutions. ("Pythagorean triangle", Uchpedgiz, 1959, §12). However, he has not led the explicit equations for solutions to this problem. Therefore, we believe that it makes sense to bring below it's a solution different from the mentioned, with the corresponding equations and give the value of the elements of the second triangle of the required type. 


\section{$\S 2$}

2.1. Firstly, we make one comment.

Let

$$
x_{i+1}, y_{i+1}, z_{i+1}, a_{i+1}, b_{i+1}
$$

are any integer solutions of the equation

$$
\mathrm{x}_{i+1}^{2}+y_{i+1}^{2}=z_{i+1}^{2}
$$

, where

$$
\left\{\begin{array}{c}
\mathrm{x}_{i+1}+y_{i+1}=a_{i+1}^{2} \\
z_{i+1}=b_{i+1}^{2}
\end{array}\right.
$$

Since, for the Pythagorean equation we have

$$
\begin{gathered}
z_{i+1}^{2}-\left(x_{i+1}+y_{i+1}\right)^{2}=\left(x_{i+1}-y_{i+1}\right)^{2} \\
2 b_{i+1}^{4}-a_{i+1}^{4}=\left(x_{i+1}-y_{i+1}\right)^{2}[2]
\end{gathered}
$$

If

$$
q_{i+1}=\left|\sqrt{\frac{z_{i+1}}{x_{i+1}+y_{i+1}}}\right|=\left|\frac{b_{i+1}}{a_{i+1}}\right|
$$

Then,

$$
\sqrt{2 q_{i+1}^{4}-1}
$$

in this case there will always be a rational number.

\section{Comment:}

[ Ljunggren proved that the equation

$$
u=\sqrt{2 q^{4}-1}
$$

has only two solutions in positive integers: 1 and 13 (W. Sierpiński,” 250 задач по элементарной теории чисел” Просвещение, Москва, 1968 р. 146). Lagrange belongs recurrence relation, through which can be found all solutions of the equation

$$
u=\sqrt{2 q^{4}-1}
$$

in rational numbers (W. Sierpiński, “О решении уравнений в целых числах”, Физматгиз, Москва, 1961, p. 80).]

Therefore, the challenge is to find all the integer values are coprime $a_{i+1}$ and $b_{i+1}$, which are then given [1] and [2] (become known value $x+y$ and $x-y$ ) allow to obtain the required values of all uniquely $x_{i+1}$ and $y_{i+1}$ in a positive integer (natural) are coprime numbers. 
2.2. In the Pythagorean triple

$$
x=m^{2}-n^{2} ; y=2 m n ; z=m^{2}+n^{2} .
$$

Let

$$
\begin{gathered}
m=m_{1}+n_{1}^{2} ; n=2 m_{1}\left(m_{1}-n_{1}\right) ; \\
m_{1}=\vartheta q ; n_{1}=\vartheta q-1
\end{gathered}
$$

then we obtained using the identity:

$$
\left[m_{1}^{2}+n_{1}^{2}+2 m_{1}\left(m_{1}-n_{1}\right)\right]^{2}-2\left[2 m_{1}\left(m_{1}-n_{1}\right)\right]^{2} \equiv\left[\left(m_{1}-n_{1}\right)^{2}-2 m_{1}^{2}\right]^{2}
$$

, we have

$$
\begin{gathered}
x+y=a^{2}=\left(1-2 \vartheta^{2} q^{2}\right)^{2} ; z=b^{2}=\left(q^{2}+\vartheta^{2}\right)^{2} ; \\
\left(2 q^{2}+1\right) \vartheta^{2}-2 q \vartheta-\left(q^{2}-1\right)=0
\end{gathered}
$$

and we obtain one of the variants of the recurrence methods for all solutions of the equations

$$
2 q^{4}-1=u^{2}
$$

and

$$
1-2 \vartheta^{2}=\gamma^{2}
$$

in the rational coprime integers:

$$
q_{i+1}=\frac{q_{i}^{2}+\vartheta_{i}^{2}}{1-2 q_{i}^{2} \vartheta_{i}^{2}}=\left|\frac{b_{i+1}}{a_{i+1}}\right| ;[3]
$$

(If $q_{i}$ and $\vartheta_{i}$ are fractional numbers, then $b_{i+1}$ equal to the numerator of the fraction as described numerator, but $a_{i+1}$ equal to the numerator of the fraction as the denominator described);

$$
\vartheta_{i+1}=\left|\frac{\left|q_{i}\right| \pm \sqrt{2 q_{i}^{4}-1}}{2 q_{i}^{2}+1}\right|[4]
$$

( $i=0,1,2,3 \ldots$. with the initial values $\left.q_{0}=1 ; \vartheta_{0}=0\right)$,such that, all integers that are coprime values $a_{i+1}$ and $b_{i+1}$ :

\section{Comment:}

[The recurrence method of Lagrange could be obtained in a simple substitution [3] value $\vartheta_{i+1}$ in [4] only using other notation

$\left(u=q_{i+1}, u_{1}=q_{i} ; \vartheta_{1}=u\right)$ :

$$
\pm u=\frac{u_{1}^{2}\left(2 u_{1}^{2}+1\right)^{2}+\left(u_{1} \pm \vartheta_{1}\right)^{2}}{\left(2 u_{1}^{2}+1\right)^{2}-2 u_{1}^{2}\left(u_{1} \pm \vartheta_{1}\right)^{2}}
$$

- The recurrence method of Lagrange for solution of equation

$$
2 u^{4}-1=\vartheta^{2}
$$

in rational numbers.] 


$$
\begin{gathered}
q_{1}=\frac{1^{2}+0^{2}}{1-2 \times 1^{2} \times 0^{2}}=\frac{1}{1} ; \vartheta_{1}=\frac{2}{3} ; \vartheta_{1}^{\prime}=0 \\
a_{1}=1 ; b_{1}=1 ; x_{1}=1 ; y_{1}=0 ; z_{1}=1^{2} ; x_{1}+y_{1}=1^{2} . \\
q_{2}=\frac{1^{2}+\left(\frac{2}{3}\right)^{2}}{1-2 \times 1^{2} \times\left(\frac{2}{3}\right)^{2}}=\frac{13}{1} ; \vartheta_{2}=\left|\frac{84}{113}\right| ; \vartheta_{2}^{\prime}=\left|\frac{2}{3}\right| ; \\
a_{2}=1 ; b_{2}=13 ; x_{2}=120 ; y_{2}=-119 ; z_{2}=13^{2} ; x_{2}+y_{2}=1^{2} . \\
q_{3}=\frac{13^{2}+\left(\frac{2}{3}\right)^{2}}{1-2 \times 13^{2} \times\left(\frac{2}{3}\right)^{2}}=\left|\frac{1525}{1343}\right| ; \vartheta_{3}=\left|\frac{84}{113}\right| ; \vartheta_{3}^{\prime}=\left|\frac{2}{3}\right| . \\
a_{3}=1343 ; b_{3}=1525 ; x_{3}=2276953 ; y_{3}=-473304 ; \\
q_{4}=\frac{z_{3}=1525^{2} ; x_{3}+y_{3}=1343^{2} .}{1-2 \times 13^{2} \times\left(\frac{84}{113}\right)^{2}}=\left|\frac{2165017}{2372159}\right| ; \vartheta_{4}=\left|\frac{6214}{57123}\right| ; \vartheta_{4}^{\prime}=\left|\frac{151245528}{262621633}\right| .
\end{gathered}
$$

The values $x_{4}, y_{4}, z_{4}$ given at the beginning of our article.

$$
\begin{gathered}
q_{5} \approx|4,3| ; q_{6} \approx|1,3| ; \\
q_{7}=\frac{\left(\frac{2165017}{2372159}\right)^{2}+\left(\frac{6214}{57123}\right)^{2}}{1-2\left(\frac{2165017}{2372159}\right)^{2}\left(\frac{6214}{57123}\right)^{2}} \approx|0,86180424|, \\
\vartheta_{7} \approx|0.4310936| ; \vartheta_{7}^{\prime} \approx|0,20176074| ; \\
q_{8} \approx|2,6| ; q_{9} \approx|1,6| ;
\end{gathered}
$$

$q_{10} \approx|0,8743101|$ and etc.

2.3. For all coprime solutions of the problem in natural numbers is a necessary and sufficient condition, that in [3]

$$
q_{i+1}=\frac{b_{i+1}}{a_{i+1}}<1
$$

(Hypotenuse trivial could be less than the sum of the two legs).

2.4. We have in [3] $q<1$ and choosing the lowest absolute value of two of its corresponding from [4] $\vartheta$ and $\vartheta^{\prime}$, we finally obtain the following $q<1$ using the method:

$$
q_{3 S+1}=\left|\frac{q_{3 S-2}^{2}+\vartheta_{3 S-2}^{2}}{1-2 q_{3 S-2}^{2} \vartheta_{3 S-2}^{2}}\right|<1[\mathbf{5}]
$$


,where $S=2,3,4, \ldots$ and etc.

\section{Comment:}

[For two integers $q_{i}=1$ and $q_{i}=13>1$ we get the maximum value from two by absolute value $\left.\vartheta_{i}.\right]$

It follows that in [5]:

2.4.1. Indeed,

$$
\begin{gathered}
q_{3 S-2}<1 \\
q^{2}+\vartheta^{2}<\left|1-2 \vartheta^{2} q^{2}\right| \\
q<\left|\sqrt{\frac{1-\vartheta^{2}}{1+2 \vartheta^{2}}}\right|
\end{gathered}
$$

and

$$
1-\vartheta^{2}<1+2 \vartheta^{2}
$$

, since otherwise there will be $3 \vartheta^{2}<0$, if $\vartheta$ is rational then is impossible.

2.4.2. It must be for the same reasons

$$
\vartheta<\left|\sqrt{\frac{1-q^{2}}{1+2 q^{2}}}\right|
$$

For example,

$$
\begin{gathered}
\vartheta_{7}=0,4310936>0,25, \\
\vartheta_{7}^{\prime}=0,20176074<0,25 .
\end{gathered}
$$

2.4.3. For $q<1$ from two

$$
\vartheta=\left|\frac{q \pm \sqrt{2 q^{4}-1}}{2 q^{2}+1}\right|
$$

one of them must be

$$
\begin{gathered}
\left|\frac{q \pm \sqrt{2 q^{4}-1}}{2 q^{2}+1}\right|<\left|\sqrt{\frac{1-q^{2}}{2 q^{2}+1}}\right| \\
2 q^{4}-q^{2}-1<0 ; q_{1}^{2}<1 ; q_{2}^{2}<-\frac{3}{4}
\end{gathered}
$$

and $q_{1}<|1|$. 
To conclude the proof, it remains to note with respect to [5] that recurrence relations is unbounded $q<1$, this means that we obtain an infinite set of Pythagorean triples whose hypotenuse and the sums of the legs are squares, and with respect to [3] and [5] the sequence gives all the solutions of the problem. Arguing as above, this completes the proof totally.

\section{Comment:}

[In the relation [5] $q_{3 s+1}$ does not depend on one, but two parameters $\left(q_{3 s-2}, \vartheta_{3 s-2}\right)$, included in this equation. And if $q_{3 s-2}<1$ is a necessary and sufficient condition for the unique determination of required values $x_{3 s-2}, y_{3 s-2}, z_{3 s-2}$, then for the next value $q_{3 s+1}<1$ this condition is only necessary, but with

$$
\vartheta_{3 s-2}<\left|\sqrt{\frac{1-q_{3 s-2}}{2 q_{3 s-2}+1}}\right|
$$

would be a necessary and sufficient.]

2.5. The second solution of the problem in natural numbers corresponding to $q_{7}$ which is defined below:

$$
\begin{gathered}
x_{7}=214038981475081188634947041892245670988588201 ; \\
y_{7}=109945628264924023237017010068507003594693720 ; \\
z_{7}=240625698472667313160415295005368384723483849= \\
=(15512114571284835412957)^{2} ; \\
x_{7}+y_{7}=323984609740005211871964051960752674583281921= \\
=(17999572487701067948161)^{2} ;
\end{gathered}
$$

\section{$\S 3$}

It follows that we have identities:

$$
\begin{aligned}
& \left(2 q^{2}+1\right)^{4}-2\left(-q \pm \sqrt{2 q^{4}-1}\right)^{4} \equiv \\
\equiv & {\left[\left(2 q^{2}-1\right) \sqrt{2 q^{4}-1} \pm 4 q\left(q^{2}+1\right)\right]^{2} } \\
& 2\left(\vartheta \pm \sqrt{1-2 \vartheta^{4}}\right)^{4}-\left(2 \vartheta^{2}-1\right)^{4} \equiv \\
\equiv & {\left[\left(2 \vartheta^{2}+1\right) \sqrt{1-2 \vartheta^{4}} \pm 4 \vartheta\left(1-\vartheta^{2}\right)\right]^{2} }
\end{aligned}
$$

for arbitrary $q$ and $\vartheta$.

With initial values $q_{0}=1$, or $\vartheta_{0}=0$ as defined above identities are transformed into recurrence equations for all rational solutions of the equations

$$
2 q_{i}^{4}-1=u^{2} ; 1-2 \vartheta_{i}^{4}=\varphi^{2}
$$

, as well as all solutions in positive integers equations

$$
\alpha^{4}-2 \beta^{4}=\gamma^{2} ; 2 r^{4}-t^{4}=k^{2} .
$$




\section{$\S 4$}

Assume that it makes sense to give these two analogies of Fermat's problem:

4.1.

$$
x^{2}+y^{2}=z^{2} ; x+y=a^{2} ; x=b^{2} .
$$

Using the same notation and procedure as in the previous case, we come to one embodiment of the recurrence methods for solutions of the equation

$$
q^{4}+\left(q^{2}-1\right)^{2}=\theta^{2}
$$

in the rational numbers:

$$
q_{i+1}=\left|\frac{q_{i}^{2}-\vartheta_{i}^{2}}{1-2 q_{i}^{2} \vartheta_{i}^{2}}\right| ; \vartheta_{i+1}=\left|\frac{q_{i} \pm \sqrt{q_{i}^{4}+\left(q_{i}^{2}-1\right)^{2}}}{2 q_{i}^{2}-1}\right|
$$

$\left(i=1,2,3 \ldots\right.$ for $\left.q_{0}=0 ; \vartheta_{0}=0 ;\right)$.

Similarly,

$$
\begin{gathered}
x_{i+1}+y_{i+1}=a_{i+1}^{2}=\left(1-2 q_{i}^{2} \vartheta_{i}^{2}\right)^{2} \\
x_{i+1}=\left(q_{i}^{2}-\vartheta_{i}^{2}\right)^{2}=b_{i+1}^{2}
\end{gathered}
$$

and

$$
q_{i+1}=\left|\frac{b_{i+1}}{a_{i+1}}\right|=\left|\sqrt{\frac{x_{i+1}}{x_{i+1}+y_{i+1}}}\right|
$$

and, therefore, the integer values $a_{i+1}$ and $b_{i+1}$.

For $q_{0}=0 ; \vartheta_{0}=0$

$$
\begin{gathered}
a_{1}=|1| ; b_{1}=0 ; x_{1}=0^{2} ; y_{1}=1 ; z_{1}=1 ; x_{1}+y_{1}=1^{2} ; \\
q_{2}=\left|\frac{0^{2}-1^{2}}{1-2 \times 0^{2} \times 1^{2}}\right|=|1| ; \vartheta_{2}=0 ;\left|\vartheta_{2}^{\prime}\right|=2 ; \\
a_{2}=1 ; b_{2}=1 ; x_{2}=1^{2} ; y_{2}=0 ; z_{2}=1 ; x_{2}+y_{2}=1^{2} \\
q_{3}=\left|\frac{1^{2}-2^{2}}{1-2 \times 1^{2} \times 2^{2}}\right|=\left|\frac{3}{7}\right| ;\left|\vartheta_{3}\right|=2 ;\left|\vartheta_{3}^{\prime}\right|=\frac{20}{31} \\
a_{3}=7 ; b_{3}=3 ; x_{3}=3^{2} ; y_{3}=40 ; z_{3}=41 ; x_{3}+y_{3}=7^{2} ;
\end{gathered}
$$

and etc. 
4.2. The second analogy of Fermat's problem:

$$
x^{2}+y^{2}=z^{2} ; x+y=a^{2} ; y=b^{2}
$$

To solve the problem could be to use the previous method, but we use the other. If

$$
x_{x}^{2}+y_{x}^{2}=z_{x}^{2}, x_{x}=b_{x}^{2} ; x_{x}+y_{x}=a_{x}^{2}
$$

,then

$$
\begin{gathered}
x_{y}=z_{x}^{4}-4 a_{x}^{4} b_{x}^{4}, y_{y}=4 a_{x}^{2} b_{x}^{2} z_{x}^{2}, z_{y}=z_{x}^{4}+4 a_{x}^{4} b_{x}^{4} \\
x_{y}^{2}+y_{y}^{2}=z_{y}^{2} ; y_{y}=b_{y}^{2}=\left(2 a_{x} b_{x} z_{x}\right)^{2} \\
x_{y}+y_{y}=\left(a_{x}^{4}-2 b_{x}^{4}\right)^{2}=a_{y}^{2}
\end{gathered}
$$

For

$$
\begin{gathered}
x_{x}=3^{2} ; y_{x}=40 ; z_{x}=41 ; a_{x}=7 ; b_{x}=3 ; \\
x_{y}=41^{4}-4(21)^{4}=2047837 ; \\
y_{y}=2^{2} 41^{2} 3^{2} 7^{2}=1722^{2}=2965284=b_{y}^{2} ; \\
z_{y}=41^{4}+4 \times 21^{4}=3603685 ; \\
x_{y}+y_{y}=5013121=2239^{2}=a_{y}^{2} .
\end{gathered}
$$

\section{References:}

[1] Diophantus of Alexandria “Arithmetica”, Наука, Главная редакция физикоматематической литературы, Москва, 1974.

[2] W. Sierpiński, “Пифагоровы треугольники”, Государственное учебно- педагогическое издательство, Москва, 1959.

[3] W. Sierpiński, “250 задач по элементарной теории чисел”, “Просвещение”, Москва, 1968.

[4] W. Sierpiński, “О решении уравнений в целых числах”, Физматгиз, Москва 1961. 